\title{
Effectiveness and quality analysis of methods in studies for the treatment of COVID-19
}

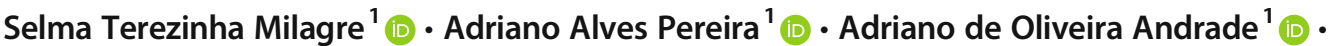 \\ Angélica de Andrade Palis ${ }^{1}$ - Ariana Moura Cabral ${ }^{1}$ (D) - Cassiana Gabriela Lima Barreto ${ }^{1}$ (D) \\ Daniel Baldoino de Souza ${ }^{1}$ (D) - Fernanda de Paula Silva ${ }^{1}$ (D) - Fernando Pasquini Santos ${ }^{1}$ (D) - Gabriella Lelis Silva ${ }^{1}$ (D) \\ José Flávio Viana Guimarães ${ }^{1}$ (D) - Laureane Almeida Santiago de Araújo ${ }^{1}$ (D) - Lígia Reis Nóbrega ${ }^{1}$ (D) \\ Luanne Cardoso Mendes ${ }^{1}$ (D) - Mariana Ribeiro Brandão ${ }^{2}$ (D) - Verônica de Lima Gonçalves ${ }^{1}$ (D) \\ Victor Hugo de Freitas Morales ${ }^{2}$ (D) $\cdot$ Viviane da Conceição Lima ${ }^{1}$ (D)
}

Received: 15 June 2020 / Accepted: 8 March 2021 / Published online: 18 March 2021

(C) Sociedade Brasileira de Engenharia Biomedica 2021

\begin{abstract}
Purpose COVID-19 has spread and continues to spread rapidly across the world. There is still no approved and effective treatment for the disease. Several drugs are being administered empirically to patients, based on results from studies with limited methodologies. There is an urgent need for systematic reviews to support decision makers and the development of guidelines. The purpose of this paper was to conduct a systematic review of drug studies carried out worldwide for COVID-19, verifying the effectiveness and methodological quality.

Method The search for relevant articles was performed in the Cochrane Central, MEDLINE, and Embase databases. The terms and combinations used for the search were ((coronavirus OR "2019-nCoV" OR 2019nCoV OR nCoV2019 OR "nCoV-2019" OR “COVID-19” OR COVID19 OR "HCoV-19” OR HCoV19 OR CoV OR "2019 novel*” OR Ncov OR "n-cov” OR "SARSCoV-2” OR "SARSCoV-2” OR “SARSCoV2" OR "SARSCoV2” OR SARSCov19 OR "SARS-Cov19” OR "SARS-Cov-19”) OR "severe acute respiratory syndrome*” OR ((corona* OR corono*) AND (virus* OR viral* OR virinae*)) AND ((treatment) OR (medication) OR (symptoms)) AND (drugs)). The review process of the articles was carried out independently by four reviewers using the PRISMA-P method.

Results As a result, a total of 30 articles were selected, which addressed the study of one or more medications for severe cases of COVID-19, which were hydroxychloroquine (or chloroquine) and azithromycin, lopinavir/ritonavir, lopinavir/ritonavir, arbidol, and Shufeng Jiedu Capsule, Combinations: remdesivir/lopinavir/homoharringtonine/emetine, remdesivir, interferon, lopinavir and ritonavir, thalidomide/methylprednisolone, tocilizumab, antimicrobial therapy.

Conclusion Despite efforts and studies, research does not provide evidence for the use of any drug to treat COVID-19, as the effects, safety, and efficacy are still uncertain. Thus, there is a need for systematic reviews to monitor, synthesize, and update the literature data on the subject, generating reliable evidence for decision makers. COVID-19 should be monitored and the need to develop effective drugs and vaccines to prevent disease progression must be reinforced.
\end{abstract}

Keywords Pandemic $\cdot$ COVID-19 $\cdot$ SARS-CoV-2 $\cdot$ Medicines

Adriano Alves Pereira aalvespereira664@gmail.com

1 Centre for Innovation and Technology Assessment in Health, Faculty of Electrical Engineering, Federal University of Uberlândia, Uberlândia, Brazil

2 Institute of Biomedical Engineering, Federal University of Santa Catarina, Florianopolis, Brazil

\section{Introduction}

According to the World Health Organization (WHO), acute respiratory diseases (ARI) occur in both the upper and lower airways, ranging from simple asymptomatic or a mild infection to a fatal disease, depending on the pathogen that caused it and also on environmental and host factors. Typically, the time of onset of symptoms after acute respiratory infection (ARI) can vary from hours to days. Common symptoms of 
ARI include fever, cough, sore throat, runny nose, shortness of breath, and wheezing. ARI can be severe, in this case, called severe acute respiratory infection (ARIS) and, in some cases, may be prone to the epidemic as is the case of severe acute respiratory syndrome (SARS).

The pathogen causing SARS was identified as a coronavirus, determined by sequencing its genome, and was designated as SARS-CoV (Yeh et al. 2004). Influenza virus and coronavirus of severe acute respiratory syndrome (SARS-CoV) are pathogens that cause ARI (World Health Organization 2014).

Currently, there is great concern about human infections caused by a coronavirus. Several viral epidemics, including severe acute respiratory syndrome (SARS-CoV) from 2002 to 2003 and H1N1 influenza in 2009, have been recorded in the past 20 years. In 2012, Middle East respiratory syndrome coronavirus (MERS-CoV) was first identified in Saudi Arabia (Cascella et al. 2020; Meo et al. 2020). SARS-CoV and MERS-CoV caused severe pneumonia, leading to the death of 774 and 858 people and with 8700 and 2494 infections, respectively, in addition to causing profound economic losses (Contini et al. 2020).

The spread of the SARS epidemic showed significant regional differences in the distribution of cases, indicating direct or indirect relationships between environmental, social, and economic factors, among others (Hu et al. 2013). The MERS outbreak is still present in some parts of the world and at the end of 2019 a new coronavirus, which causes the disease called COVID-19 appeared in Wuhan, Hubei Province in China (Contini et al. 2020). As a result, the population now lives with the prevalence of old outbreaks, such as MERS, and with the emergence of a new pandemic caused by COVID-19.

COVID-19 is a disease caused by a beta coronavirus called SARS-CoV-2 (severe acute respiratory syndrome type 2 coronavirus). Just as the other CoVs are sensitive to ultraviolet rays and heat and "can be effectively inactivated by lipid solvents, including ether (75\%), ethanol, disinfectants containing chlorine, peroxyacetic acid and chloroform, except chlorhexidine" (Cascella et al. 2020). The World Health Organization (WHO) classified the threat to the $\mathrm{CoV}$ epidemic as a "very high level" on 28 February 2020. The WHO declared COVID19 a pandemic when, on 11 March, the number of countries presenting the disease tripled and the number of cases increased 13 times outside of China (Cascella et al. 2020).

Human history reveals to us several pandemics whose frequency seems to grow as the emergence of viral diseases in animals increases. Most of the new pandemics originated through the transmission of pathogens from animals (pigs, birds, and wild animals) to humans. Thus, the world has experienced several pandemics such as the black plague, Spanish flu, and acquired immune deficiency syndrome (AIDS). A pandemic can result in sudden and generalized morbidity and mortality and also lead to social, political, and economic conflicts (Madhav et al. 2017).
COVID-19 is a zoonotic virus, and phylogenetic analysis shows bats as a reservoir for the virus. However, the intermediate host has not yet been identified and there are suspicions of an exotic animal called Pangolin. The main form of transmission of the new coronavirus occurs during contact, without protection, with the infected person, who transmits the virus through droplets and fomites (World Health Organization (WHO) 2020). According to van Doremalen et al. (2020), the SARS-COV-2 can remain viable and infectious for several hours in aerosols and even for days on surfaces such as stainless steel, cardboard, plastic, and copper, being more stable in stainless steel and plastic than in copper and cardboard. Thus, it also propagates when the person touches a contaminated surface or object and then on the eyes, nose, or mouth. According to Paudel et al. (2020), the mean incubation period was 5 days, with an interval of 1 to 14 days, $95 \%$ of patients presented symptoms in the interval of 11 to 12 days after contact, and it was observed that it is possible to find asymptomatic carriers with an incubation period of 19 days.

Following the symptoms of SARS, COVID-19 has a broad spectrum of clinical manifestation and causes flu-like breathing problems with symptoms such as cough, fever, and, in more severe cases, breathing difficulties (it is estimated that up to $6 \%$ of hospital admissions progress to SARS and require care in intensive care units (ICU) (World Health Organization (WHO) 2020)). With confirmed cases of COVID-19 exceeding 41 million worldwide and more than 1 million deaths (OPAS/OMS Brasil 2020), scientists around the world are working to find ways to slow the spread of the new coronavirus and find effective treatments.

Several studies on treatments and vaccines against COVID-19 are advancing and new research is being added on a daily basis. Treatments are being proposed for people with COVID-19 in studies that attempt to demonstrate the effectiveness of antiviral treatments, based on previous interventions to treat other diseases such as Influenza, MERS, Ebola, HIV/AIDS, and malaria treatments that were first developed decades ago, among others already existing for different pathologies (Liu et al. 2020a).

In addition to antivirals, other treatments are being proposed using immunomodulators (i.e., interferon and tocilizumab), corticosteroids, and polyclonal antibodies, besides the generic viral treatments such as vitamin C, zinc, and selenium (Juul et al. 2020; Singh et al. 2020). These treatments are being administered to patients on an empirical basis (Liu et al. 2020c).

Despite all studies, WHO has not yet found a treatment for COVID-19, but recommends supportive treatment according to the needs of each patient and indicates that in some cases empirical antimicrobial therapy may be given to the patient (Tobaiqy et al. 2020). Therefore, the inclusion or exclusion of potential treatments for COVID-19 is essential for the safety of the population. A systematic review allows us to synthesize the evidence, thereby reducing the time from evidence to clinical practice. 
Further, considering that COVID-19 can impair the function of several organs, including significantly affecting cardiac function (Inciardi et al. 2020), a systematic review of the drugs used and the quality of studies is important to assist in the development of treatment protocols.

At this moment, treatment protocols based on fragile evidence can lead to serious results for the patient, including death. Public health policies must be based on the best evidence available to prevent, treat symptoms, cure, and intervene in the consequences of COVID-19. In this scenario, our objective was to systematically review the literature and verify the efficacy and quality of the evidence of the drugs, through the methodology used, in the treatment of patients with COVID-19 to assist in the recommendations of evidence-based guidelines for the treatment of COVID-19 (Das et al. 2020).

\section{Methods}

This systematic review was developed according to the Preferred Reporting Items for Systematic Reviews and Meta-Analysis method (PRISMA-P) (Moher et al. 2016). Our team is made up of Doctors and Biomedical Engineers to ensure expertise in a number of areas.

\section{Study design}

A systematic review of retrospectives and prospectives studies, following the guidelines of PRISMA-P. The entire process of selecting the studies was carried out by four reviewers and summarized in a PRISMA flow diagram (Fig. 1).

\section{Inclusion/exclusion criteria}

Eligible articles accounted for studies with respiratory diseases treatments, including MERS and SARS, but focused on COVID-19. Due to the lack of randomized controlled trials, the authors also included epidemiological studies, cross-sectional studies, case studies, clinical observations, health organ reports, prospective cohort, case-control studies, systematic reviews, meta-analyses, and non-randomized studies associated with COVID-19. The research included all languages and was limited to articles published in 2020. A bibliographic review was conducted between March and April 2020.

The study designs studied were, and randomized clinical trials published on respiratory diseases, including MERS and SARS, and mainly on COVID-19. There were no restrictions on the language and year of publication.

\section{Search sources}

Three databases were consulted for evidence referring to the drugs indicated for COVID-19 (Cochrane Central,
MEDLINE, and Embase) (Singh et al. 2020). For this systematic review, the following terms and combinations were used (Singh et al. 2020):

(coronavirus OR "2019-nCoV” OR 2019nCoV OR nCoV2019 OR “nCoV-2019” OR “COVID-19” OR COVID19 OR "HCoV-19” OR HCoV19 OR CoV OR "2019 novel*" OR Ncov OR "n-cov" OR "SARS-CoV-2" OR "SARSCoV-2" OR "SARSCoV2" OR "SARSCoV2" OR SARSCOv19 OR “SARS-Cov19” OR “SARS-Cov-19”) OR "severe acute respiratory syndrome*" OR ((corona* OR corono*) AND (virus* OR viral* OR virinae*)) AND ((treatment) OR (medication) OR (symptoms)) AND (drugs).

The title and abstract of the articles were analyzed by four examiners.

\section{Medicines included and outcomes}

Our criteria for including medicines and outcomes were unrestricted, however, specific to treatments on COVID-19.

\section{Selection}

Four authors (AAP, AOA, FPS, and STM) independently analyzed the titles and abstracts of the studies identified by the research strategy and verified the existing duplicates to remove them. Eligible studies were reevaluated by reading the full text to compose this systematic review. In case of disagreement in the chosen articles, the opinion of a fifth examiner was requested. However, no study required consultation with a fifth reviewer.

The flowchart in Fig. 1 shows the selection process of the study.

\section{Data acquisition}

Two authors (JFVG and VHFM) extracted data from the selected articles and two other authors verified the accuracy and fidelity of the data presented and another author resolved the existing disagreements.

\section{Assessment of bias quality and risk of included studies}

No methodological quality assessment tools have been used for this study, given that the results of studies on the effects of the SARS-CoV-2 pandemic are still preliminary, given the recent and abrupt global impact of this health emergency on public health services, it is currently not possible to assess the methodological quality of studies (Juul et al. 2020). However, the following items were analyzed: 
Fig. 1 Flow Chart of the search process

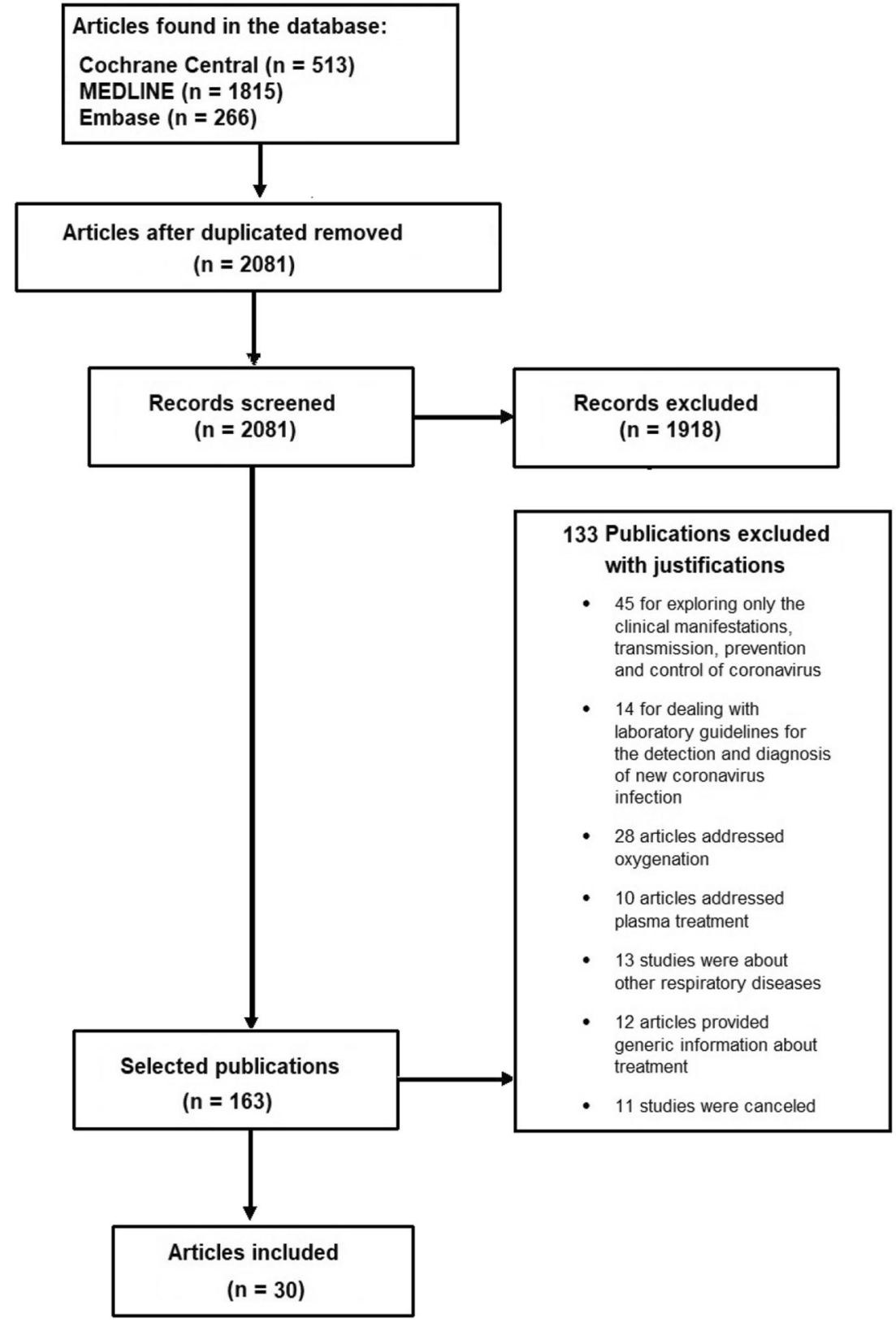

- All articles that presented treatment with medications, but COVID-19 was not the main focus of the study and was excluded.

- All articles that caused doubts about the inclusion in this systematic review were re-analyzed for relevance.

- Studies with failure to follow up above $10 \%$ were excluded.

\section{Results}

The search in the databases resulted in a total of 2594 articles that had in their titles, or in the abstracts, and the search terms.
The duplicates were ignored and 2081 articles remained. After the analysis of titles and abstracts, 163 articles were selected.

After reading the full text of these 163 articles, 133 articles were excluded, 45 for exploring only the clinical manifestations, transmission, prevention, and control of coronavirus, 14 for dealing with laboratory guidelines for the detection and diagnosis of new coronavirus infection, 28 articles addressed oxygenation, 10 articles addressed plasma treatment, 13 studies were about other respiratory diseases, 12 articles provided generic information about treatment, and 11 studies were canceled.

As a result, 30 articles were eligible for this systematic review, which presents one or more drugs that are being used experimentally in other countries to combat the COVID-19 pandemic. 
The studies were grouped according to the methods used, as shown in Table 1.

Table 2 shows the number of studies included in the systematic review concerning the drug.

Table 3 shows the result of the articles in relation to the effectiveness of the drugs.

\section{Discussion}

There is no specific medicine to treat or prevent SARS-CoV-2 until this time. However, a number of clinical studies are being conducted in the search for an effective substance. In view of the global pandemic and the severity of the disease, health professionals are using medicines that have previously been effective in similar diseases, such as SARS and MERS, and have proven to be effective.

Table 1 shows the 30 articles that were used for this systematic review with the names of the authors and the drugs used. From Table 1, the medicines were grouped according to the type and combinations and shown in Table 2. The data in Table 2 indicate that despite the number of articles found, the studies are based on only 9 different medicines, shown in Fig. 2.

It can be seen from Fig. 2 that the studies found were more focused on hydroxychloroquine (or chloroquine) and azithromycin (16 studies).

The following are presented the 9 medicines included in this systematic review.

\section{Medicines included in this systematic review}

\section{Hydroxychloroquine (or chloroquine) and azithromycin}

We found 16 articles related to the mutual use of hydroxychloroquine (or chloroquine) and azithromycin. (Colson et al. 2020) studied the in vitro activity of chloroquine against SARS-CoV-2, the results showed that chloroquine is effective in the treatment of pneumonia in patients with COVID-19. The authors also report that clinical trials conducted in China, with more than 100 patients, showed that chloroquine reduces hospitalization time and has positive results about pneumonia. For the authors, hydroxychloroquine has the same mechanism of action as chloroquine and can be used in the treatment of COVID-19. Cortegiani et al. (2020) conducted a systematic review on the use of chloroquine and stated that there are a preclinical justification and sufficient evidence on the efficacy of chloroquine during the treatment of COVID-19.

Deretic and Timmins (2020) conducted in vitro research on respiratory epithelial cells using azithromycin, chloroquine, and hydroxychloroquine to evaluate the effects caused by these drugs. As a result, the authors reported that azithromycin, chloroquine, and hydroxychloroquine control the $\mathrm{pH}$ of respiratory epithelial cells and reduce the bacteria's adherence to lung epithelial cells. Fantini et al. (2020) performed in silico tests and used a combination of structural and molecular modeling approaches and showed that chloroquine and hydroxychloroquine at viral peak prevent the binding of gangliosides. The authors conclude that the identification of this new mechanism of action of chloroquine and hydroxychloroquine supports the use of these drugs to cure patients infected with SARS-CoV-2, which may interrupt the pandemic.

Gao et al. (2020) from multicenter clinical analysis on chloroquine phosphate and hydroxychloroquine, according to the authors, chloroquine antiviral and anti-inflammatory activities may be responsible for the efficacy in the treatment of patients with COVID-19 pneumonia. The authors state that the results obtained from more than 100 patients showed that chloroquine phosphate is superior to standard treatment and no serious adverse reactions to chloroquine phosphate were observed in patients.
Table 1 Studies included in the systematic review

\begin{tabular}{|c|c|}
\hline Authors & Medications \\
\hline $\begin{array}{l}\text { Colson et al. (2020), Cortegiani et al. (2020), Deretic and Timmins (2020), } \\
\text { Fantini et al. (2020), Gao et al. (2020), Gautret et al. (2020), } \\
\text { Kupferschmidt and Cohen (2020), Liu et al. (2020b), Scuccimarri et al. } \\
\text { (2020), Wang et al. (2020a), Weston et al. (2020), Yao et al. (2020), } \\
\text { Geleris et al. (2020), Rosenberg et al. (2020), Mercuro et al. (2020), } \\
\text { Tang et al. (2020). }\end{array}$ & $\begin{array}{l}\text { Hydroxychloroquine (or chloroquine) } \\
\text { and azithromycin }\end{array}$ \\
\hline Cao et al. (2020), Lim et al. (2020), Li et al. (2020). & Lopinavir-ritonavir \\
\hline Wang et al. (2020b) & $\begin{array}{l}\text { Lopinavir/ritonavir, arbidol, and Shufeng } \\
\text { Jiedu Capsule }\end{array}$ \\
\hline Choy et al. (2020) & $\begin{array}{l}\text { Combinations: } \\
\text { remdesivir/lopinavir/- } \\
\text { homoharringtonine /emetine }\end{array}$ \\
\hline $\begin{array}{l}\text { Reina (2020), Wang et al. (2020a), Zhai et al. (2020), de Wit et al. 2020), } \\
\text { Holshue et al. (2020). }\end{array}$ & Remdesivir \\
\hline Liu and Gao (2020) & Interferon, lopinavir, and ritonavir \\
\hline Chen et al. (2020) & Thalidomide/methylprednisolone \\
\hline Xu et al. (2020) & Tocilizumab \\
\hline Li et al. (2020) & Antimicrobial therapy \\
\hline
\end{tabular}


Table 2 Number of studies included in the systematic medication review

\begin{tabular}{ll}
\hline Medicines & Number of related studies \\
\hline Hydroxychloroquine (or chloroquine) and azithromycin & 16 \\
Lopinavir-ritonavir & 3 \\
Lopinavir/ritonavir, arbidol, and Shufeng Jiedu Capsule & 1 \\
Combinations: remdesivir/lopinavir/homoharringtonine/emetine & 1 \\
Remdesivir & 5 \\
Interferon, lopinavir, and ritonavir & 1 \\
Thalidomide/methylprednisolone & 1 \\
Tocilizumab & 1 \\
Antimicrobial therapy & 1 \\
\hline
\end{tabular}

In their study, Gautret et al. (2020) observed 36 positive COVID-19 patients treated with hydroxychloroquine for 14 days. In addition, 6 patients in the intervention group received azithromycin to prevent bacterial infection. The main result was that on the sixth day of treatment, patients receiving hydroxychloroquine were more likely to be tested negative for the SARS-CoV-2 virus and 6 patients receiving hydroxychloroquine and azithromycin tested negative on the sixth day of treatment.

Kupferschmidt and Cohen (2020) reported possible treatments based on pre-existing diseases, highlighting the advantages and disadvantages of each treatment. In this study, chloroquine and hydroxychloroquine received greater attention due to the positive results of small studies. The medications are effective in inhibiting the virus, however, with the need for a high dosage, which can increase the risks of severe toxicity. The authors also warn that studies have shown the efficacy of treatment using hydroxychloroquine; however, these are nonrandomized studies without clinical outcomes, such as the number of deaths.

Liu et al. (2020b) evaluated the antiviral effect activities of chloroquine and hydroxychloroquine in vitro cells against the SARS-CoV-2 virus. Based on the results found, the authors found that hydroxychloroquine was less potent than chloroquine. Thus, although the results point to the efficacy of both drugs, hydroxychloroquine, besides having antiviral activity is also an anti-inflammatory agent, and may decrease the production of cytosines in cells.

Scuccimarri et al. (2020) reported experience with other diseases to demonstrate the efficacy of hydroxychloroquine. Additionally, they used recent studies of the performance of chloroquine and hydroxychloroquine in COVID-19. The authors reported that recent studies on chloroquine and hydroxychloroquine for the treatment of COVID-19 demonstrated the ability to reduce the activity of the SARS-CoV-2 virus. The activity of chloroquine and hydroxychloroquine is affected by an increase in endosomal $\mathrm{pH}$, which may block virus infection.
Wang et al. (2020a) evaluated the antiviral efficacy of chloroquine and other drugs in vitro. The results revealed that chloroquine is highly effective in controlling the SARSCoV-2 infection in vitro. Weston et al. (2020) conducted an in vitro search of Vero E6 cells, comparing them to nonSARS-CoV-2 cells. Chloroquine and hydroxychloroquine had anti-SARS-CoV-2 effects in humans, reducing the messenger RNA of the virus and a significant reduction in viral replication, both in the use of chloroquine and in the use of hydroxychloroquine. However, hydroxychloroquine showed higher antiviral activity, since the production of the virus was more sensitive to this compound, with greater inhibition observed in the same treatment concentration.

Yao et al. (2020) conducted in vitro research on SARSCoV-2 contaminated cells to evaluate the pharmacological activity of chloroquine and hydroxychloroquine using physiological pharmacokinetic models of both drugs. It was observed that hydroxychloroquine was shown to be more potent compared to chloroquine. Geleris et al. (2020) used multivariate Cox regression models to evaluate the association between hydroxychloroquine use and respiratory failure of patients diagnosed with COVID-19 in New York City. A total of 1376 patients were included in the study, of which 58.9\% (811 patients) received hydroxychloroquine treatment and $41.1 \%$ (565 patients) did not receive hydroxychloroquine. There was no statistical difference in intubation and death outcomes between the group receiving hydroxychloroquine and the group receiving standard treatment.

Rosenberg et al. (2020) tried to describe the association between clinical results in hospitalized patients with a positive diagnosis for COVID-19 and the use of hydroxychloroquine alone, isolated azithromycin, and the combined use of the two drugs. They included 1438 patients who tested positive for COVID-19 in different hospitals in the State of New York and were randomly selected. The patients were divided into 4 treatment groups: 271 patients received standard treatment and hydroxychloroquine; 735 patients received standard treatment and hydroxychloroquine combined with 
Table 3 Summary of drug efficacy

\begin{tabular}{|c|c|c|}
\hline Author & Study design & Efficacy \\
\hline \multicolumn{3}{|c|}{ Hydroxychloroquine (or chloroquine) and azithromycin } \\
\hline Colson et al. (2020) & In vitro & Yes \\
\hline Cortegiani et al. (2020) & Systematic review & Yes \\
\hline Deretic and Timmins (2020) & In vitro & Yes \\
\hline Fantini et al. (2020) & In silico & Yes \\
\hline Gao et al. (2020) & Multicenter clinical trials & Yes \\
\hline Gautret et al. (2020) & $\begin{array}{l}\text { Open-label non-randomized } \\
\text { clinical trial }\end{array}$ & Yes \\
\hline Kupferschmidt and Cohen (2020) & Report & Yes \\
\hline Liu et al. (2020b) & In vitro & Yes \\
\hline Scuccimarri et al. (2020) & Editorial & No \\
\hline Wang et al. (2020a) & In vitro & Yes \\
\hline Weston et al. (2020) & In vitro & Yes \\
\hline Yao et al. (2020) & In vitro & Yes \\
\hline Geleris et al. (2020) & Cohort & No \\
\hline Rosenberg et al. (2020) & Retrospective multicenter cohort study & No \\
\hline Mercuro et al. (2020) & cohort study & No \\
\hline Tang et al. (2020). & $\begin{array}{l}\text { Multicenter, open-label, randomized } \\
\text { controlled trial }\end{array}$ & No \\
\hline \multicolumn{3}{|l|}{ Lopinavir-ritonavir } \\
\hline Cao et al. (2020) & Randomized, controlled, open-label trial & No \\
\hline Lim et al. (2020) & Case report & Yes \\
\hline Li et al. (2020). & Specialist panel & Yes (with restrictions) \\
\hline \multicolumn{3}{|c|}{ Lopinavir/ritonavir, arbidol, and Shufeng Jiedu Capsule } \\
\hline Wang et al. (2020b) & Retrospective study & Yes \\
\hline \multicolumn{3}{|c|}{ Combinations: Remdesivir/lopinavir/homoharringtonine/emetine } \\
\hline Choy et al. (2020) & In vitro & Yes \\
\hline \multicolumn{3}{|l|}{ Remdesivir } \\
\hline Reina (2020) & Narrative review & Yes \\
\hline Wang et al. (2020a) & In vitro & Yes \\
\hline Zhai et al. (2020) & Systematic review & No \\
\hline de Wit et al. (2020) & Animal study & Yes \\
\hline Holshue et al. (2020) & Case report & Yes \\
\hline \multicolumn{3}{|l|}{ Interferon, lopinavir, and ritonavir } \\
\hline Liu and Gao (2020) & Retrospective, case series & Yes \\
\hline \multicolumn{3}{|l|}{ Thalidomide/methylprednisolone } \\
\hline Chen et al. (2020) & Case report & Yes \\
\hline \multicolumn{3}{|l|}{ Tocilizumab } \\
\hline Xu et al. (2020) & Case series & Yes \\
\hline \multicolumn{3}{|l|}{ Antimicrobial therapy } \\
\hline Li et al. (2020) & Specialist panel & Yes \\
\hline
\end{tabular}

azithromycin; 211 patients received standard treatment and azithromycin; and 221 received only standard treatment. The authors concluded that there was no statistical difference between standard treatment compared to any other treatment using hydroxychloroquine or azithromycin, as well as the association of these drugs.
Mercuro et al. (2020) conducted a retrospective cohort study and evaluated patients with COVID-19 in Boston who would have received at least 1 day of hydroxychloroquine, characterizing them for the risk and degree of prolongation of the corrected QT interval (QTc). The study included 90 patients who tested positive for the new coronavirus (SARS- 
Fig. 2 Medicines used and number of studies included in this systematic review

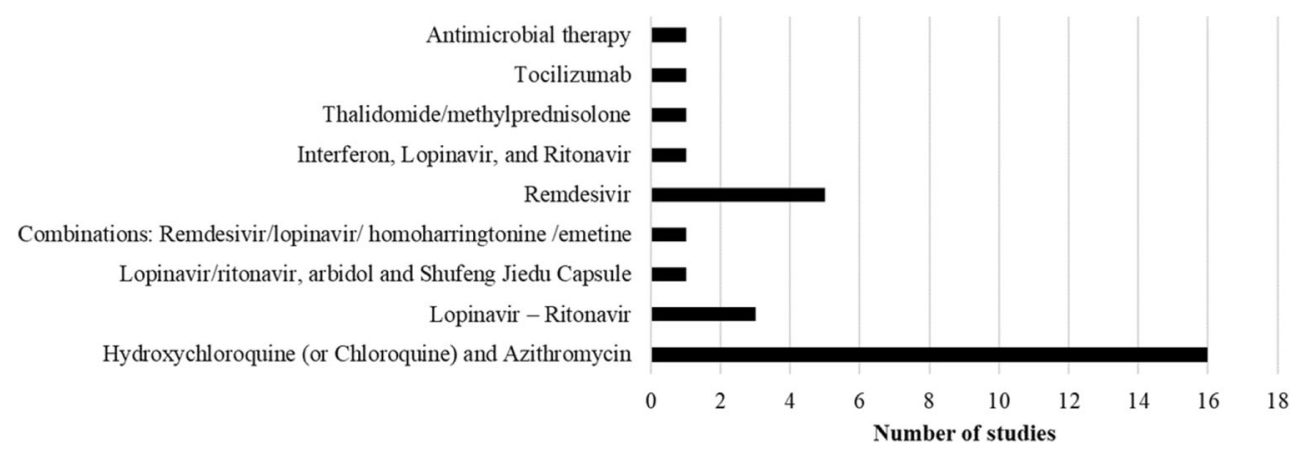

CoV-2). All received hydroxychloroquine, and 53 patients received hydroxychloroquine combined with azithromycin. The authors concluded that patients who received hydroxychloroquine for the treatment of COVID-19associated pneumonia had a high risk of QTc prolongation. Additionally, concomitant treatment of hydroxychloroquine and azithromycin had greater changes in QTc.

Tang et al. (2020) conducted a multicenter, randomized, parallel, and open clinical trial to evaluate the efficacy and safety of hydroxychloroquine in adult patients with COVID19. The study included 150 patients. Patients were randomly divided into 2 groups: standard treatment group associated with the use of hydroxychloroquine and standard treatment group. The authors concluded that the administration of hydroxychloroquine had no significant difference concerning the standard treatment; also, adverse events were higher in the group receiving hydroxychloroquine.

The largest number of articles found were those that evaluated chloroquine, hydroxychloroquine, and azithromycin, probably due to the very positive initial results. The explanations for the benefits of these drugs have been described in several ways by the authors. Gao et al. (2020) found that chloroquine blocks COVID-19 infection at low micromolar concentration. They reported that the efficacy of treatment using hydroxychloroquine and azithromycin was due to the potent efficacy of hydroxychloroquine and a potential synergistic effect of azithromycin. Liu et al. (2020b) conducted an in vitro study on the mechanisms of action of chloroquine and hydroxychloroquine in inhibiting virus entry and suggested that both blocked the transport of the SARS-CoV-2 virus from the endosomes to lysosomes. The authors suggest the association of COVID-19 severity with increased cytosine concentration in blood plasma and that hydroxychloroquine acts to decrease cytosine concentration. Sargiacomo et al. (2020) suggested that the mortality rate in patients with advanced ages is due to the high number of senescent cells. Hydroxychloroquine alkalizes the $\mathrm{pH}$ in lysosomes, which accumulate in senescent cells, preventing and the accumulation of one of the senescence markers. Azithromycin acts as a sinolytic drug and can selectively target and remove senescent cells.

\section{Lopinavir-ritonavir}

We found 3 articles referring to the joint use of Lopinavir and Ritonavir. Cao et al. (2020) conducted a randomized, controlled, and open-label study involving adult hospitalized patients with confirmed SARS-CoV-2 infection. Patients were randomly divided into a ratio of $1: 1$ to receive standard treatment associated with lopinavir-ritonavir. The authors concluded that in adult patients hospitalized with severe COVID-19, no benefit was observed with treatment with lopinavir-ritonavir, in addition to standard treatment.

Lim et al. (2020), in their case study, reported that they accompanied a 54-year-old man with COVID-19. Lopinavirritonavir was administered on the tenth day after the onset of COVID-19, with a decrease in the SARS-CoV-2 virus. The authors concluded that it is not possible to affirm that the decrease in the SARS-CoV-2 resulted from the administration of lopinavir-ritonavir, as the decrease may be due to the natural course of the healing process, or both.

Li et al. (2020) standardized diagnostic and treatment services in China. For treatment, the only antiviral recommendation is lopinavir-ritonavir with the recommended administration of 2 tablets twice daily for 14 days.

\section{Lopinavir/ritonavir, arbidol, and Shufeng Jiedu Capsule}

An article was found with studies on lopinavir/ritonavir, arbidol, and Shufeng Jiedu Capsule. Wang et al. (2020b) conducted a study with 4 patients with SARS-CoV-2-associated pneumonia. In all patients, the antivirals lopinavir-ritonavir (Kaletra®), arbidol, and Shufeng Jiedu Capsule (SFJDC, a traditional Chinese medicine) were administered. After treatment, three patients experienced significant improvement in symptoms associated with pneumonia.

\section{Combinations: \\ Remdesivir/lopinavir/homoharringtonine/emetine}

An article was found that dealt with the various combinations between remdesivir, lopinavir, homorringtonine, and emetine. This study was proposed by (Choy et al. (2020) who evaluated 
the in vitro antiviral effect of previously reported compounds to inhibit the replication of coronavirus and compounds currently under evaluation in clinical trials for patients with COVID-19: remdesivir, ribavirin, favipiravir, lopinavir, homorringtonine, and emetine. Ribavirin or favipiravir currently evaluated in clinical trials did no show inhibition of the SARS-CoV-2 virus. Synergy was observed between remdesivir and emetine, which may achieve inhibition of $64.9 \%$ in viral replication.

\section{Remdesivir}

We found 5 articles that studied remdesivir applied to the fight against COVID-19. Reina (2020) made a narrative review on antiviral drugs capable of inhibiting replication of the new coronavirus. The authors concluded that, in vitro and animals, remdesivir has a high capacity to block infections and viral replication with attainable concentrations in human plasma. The authors argue that remdesivir is one of the few antiviral drugs with proven efficacy.

Wang et al. (2020a) evaluated in vitro the antiviral efficiency of remdesivir and other medicines. The results reveal that remdesivir is highly effective in controlling the SARS-CoV-2 infection. Also, when using a human cell, the authors showed that the drug also inhibited viral infection.

Zhai et al. (2020) conducted a literature review on the epidemiology, diagnosis, isolation, and treatment of COVID-19. This review presented an article that used the drug remdesivir in monkeys (de Wit et al. 2020). The results of these studies showed improvement in pulmonary function, reduction of viral load, and impediment of lung lesions. The first case of treatment of a patient with COVID-19 with remdesivir was made in the USA (Holshue et al. 2020), with an improvement in the clinical condition in only 1 day of treatment.

\section{Interferon, lopinavir, and ritonavir}

About the use of interferon, lopinavir, and ritonavir, 1 study was found. Liu and Gao (2020) conducted a retrospective case series study in 51 patients admitted to China with COVID-19. All patients received the combination of Interferon inhalation and the antivirals lopinavir and ritonavir. In this treatment, 1 patient died and the other 50 patients were discharged from the hospital.

\section{Thalidomide/methylprednisolone}

A study was found in which thalidomide/methylprednisolone treatment was performed. Chen et al. (Chen et al. 2020) conducted a case study in China. The history of the patient in the hospital indicates that before treatment with thalidomide/ methylprednisolone there was worsening of his daily condition. No adverse reactions were verified in this study and the symptoms presented by the patient disappeared after 3 days of thalidomide administration.

\section{Tocilizumab}

It was found 1 article about tocilizumab applied in patients with COVID-19. Xu et al. (2020) evaluated the effectiveness of tocilizumab in severe patients with COVID-19. Twenty patients were analyzed. The results of the authors showed that 5 days after the beginning of the administration of tocilizumab, 15 patients had decreased the need for oxygen and 1 patient did not require oxygen therapy. No adverse reaction was observed. After 16 days, all patients were liberated from the hospital.

\section{Antimicrobial therapy}

Li et al. (2020) indicate that if the bacterial infection is suspected, according to the patient's clinical and imaging findings, patients of mild type for COVID-19 may take oral antibiotics for acquired pneumonia, such as cephalosporins or fluoroquinolones.

\section{Methodological limitations of the studies included}

The cited articles suggest medications for the treatment of COVID-19. However, most studies have to be considered with a high level of evidence. These limitations include the study design, number of patients analyzed, diseases other than COVID-19, in vitro or in silico studies, animal studies, heterogeneity between groups, inadequate randomization, effects on diseases other than COVID-19 such as SARS and MERS and, in multicenter studies, heterogeneity in the protocol used. However, the main limitation observed in the studies was blinding, which did not occur in any of the studies included in this systematic review. The main methodological limitations of the articles included in this systematic review are: the studies by Colson et al. (2020), Deretic and Timmins (2020), Liu et al. (2020b), Wang et al. (2020a), Weston et al. (2020), Yao et al. (2020) and Choy et al. (2020) were based on in vitro studies. The Systematic Review of Cortegiani et al. (2020) was based on one narrative letter, one in vitro study, one editorial, expert consensus paper, two national guideline documents, and 23 clinical studies in progress, with recruitment not complete in none of the clinical studies. The study by Fantini et al. (2020) was carried out in silico. The conclusions of Gao et al. (2020) are based on clinical studies but did not report how these studies were conducted.

Gautret et al. (2020) did not randomize patients, the study had small sample, and there were heterogeneities in terms of viral load and treatment between the two groups. Kupferschmidt and Cohen (2020) highlighted some drugs 
considering the similarity of COVID-19 with pre-existing diseases, such as SARS and MERS, without having proven scientific evidence of these drugs in relation to the new coronavirus. Although some drugs, such as hydroxychloroquine, have been shown to be effective in the treatment of COVID19 , the studies evaluated were not randomized and clinical outcomes, such as deaths, were not cited. Scuccimarri et al. (2020) carried out a systematic review based on studies with small samples, some with serious methodological flaws, with no report of the clinical outcome and a control group recruited from other centers, all articles presented at least one of the problems mentioned.

Geleris et al. (2020) conducted an observational study without randomizing the groups. Rosenberg et al. (2020) did not achieve homogeneity at the time of treatment initiation; in addition, they carried out an observational study. (Mercuro et al. 2020) did not use a control group. The study carried out by Tang et al. (2020) was not blind, randomization was not adequate, power of the test was small due to the sample size, and as it was a multicenter study, the protocol was not followed in the same way in all study centers. The study by Cao et al. (2020) is a non-blind study, and it is possible that knowledge of the attribution of treatment may have influenced clinical decision-making, the article does not provide data on lopinavir exposure levels in critically ill patients. Lim et al. (2020), Holshue et al. (2020), and Chen et al. (2020) carried out a case study.

The recommendations presented by $\mathrm{Li}$ et al. (2020) were based on the opinion of experts and scientific articles, which indicates the use of lopinavir-ritonavir. However, the articles are based on the treatment of other diseases. In the study by Wang et al. (2020b), the sample consisted of only 4 patients. Reina (2020) presents a narrative review, where all studies were performed with SARS-CoV and MERS-CoV (and not SARS-CoV-2). In addition, the studies are mostly in vitro or with animals. Zhai et al. (2020) did not present the search strategies; the literature review was performed mainly with articles evaluating drugs for the treatment of SARS-CoV and MERS-CoV, absence of scientific evidence from randomized clinical trials for the treatment of COVID-19.

The study by de Wit et al. (2020) was carried out with animals and for MERS-CoV infection; in addition, the authors have a conflict of interest, as they are employees of the company that makes the drug. Liu and Gao (2020) presented a study with a small sample size, and there was no homogeneity between the severity of COVID-19. Xu et al. (2020) presented an observational study (non-randomized and without a control group) with only 21 patients. The recommendations presented by Li et al. (2020) were based on the opinion of experts and scientific articles, which indicates the use of Antimicrobial therapy. However, the articles are based on the treatment of other diseases.

Table 3 shows the results of Table 1, organized according to the study design of each article and the efficacy of the analyzed drugs. Based on Table 3, Fig. 3 was generated, showing the study design used and the respective number of articles included in this systematic review.

Figure 3 shows that most of the studies performed were in vitro ( 8 studies), followed by cohort and case report studies (3 studies).

According to Table 3, the association of hydroxychloroquine (or chloroquine) and azithromycin was effective in 11 of the studies presented. However, 6 of these studies were conducted in vitro, 1 was performed in silico, 1 systematic review that included articles with various methodological problems, 1 report based on multicenter clinical trials without explanation of the research protocol, and 1 open-label non-randomized clinical study trial that produced low evidence due to the adopted methodology. The studies that do not prove the efficacy of the association hydroxychloroquine (or chloroquine) and azithromycin are more solid, but also do not generate strong evidence, since most of them were not blind, controlled nor randomized studies.

Fig. 3 Study designs and respective study quantities included in this systematic review

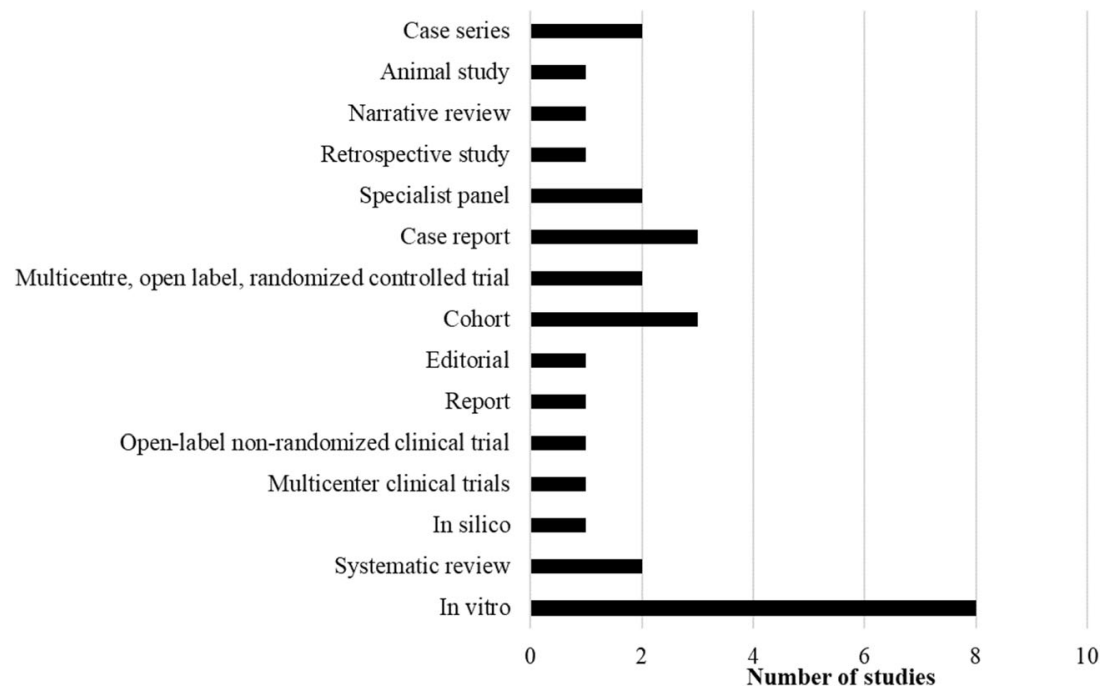


The efficacy in relation to lopinavir/ritonavir antivirals was confirmed by 2 studies, 1 of these articles was a case report and 1 article composed by a group of experts that adds reservations regarding the effectiveness of antivirals in general. Also, in this case, the more solid study does not prove the use of lopinavir/ ritonavir drugs for the treatment of COVID-19.

In the case of the combination of the drugs lopinavir/ritonavir, arbidol, and Shufeng Jiedu Capsule, only 1 article was found, which is effective in the treatment of COVID-19 for these drugs. However, it is a case series study with only 4 patients, which is a small sample to generate evidence.

For the combinations remdesivir/lopinavir/homo harringtonine/emetine, the article found reports the effectiveness of the combination, but the study was carried out in vitro. In relation to remdesevir, 5 studies were found; in four of these articles, the results show the effectiveness of the treatment using remdesivir, 1 of the articles presented a narrative review, 1 article used an in vitro study, 1 article presented an animal study, and 1 study was of a case report. However, the study with more elaborate methods did not show the effectiveness of remdesivir.

The researchers found 1 paper for each of the drugs interferon/lopinavir/ritonavir, thalidomide/methylprednisolone, tocilizumab, and antimicrobial therapy. All the studies found showed results showing the effectiveness of these drugs, but the studies have a low level of evidence due to the methodology used.

\section{Conclusion}

The main studies included in this systematic review were based on the treatment of pre-existing diseases similar to the new coronavirus and in vitro studies, preventing support for any decision based on scientific evidence.

Although efforts have been made in research related to drugs for COVID-19, the available evidence does not provide safety for its use, as the effects, safety, and efficacy are still uncertain. It was found that the studies conducted so far on the drugs for the treatment of COVID-19 lack greater scientific rigor, especially the studies that had a positive outcome for chloroquine, hydroxychloroquine, and azithromycin, since the majority did not have the control group and, in the presence of a control group, there was no randomization, besides other problems such as the small size of the groups.

Although some drugs have a positive response in the fight against SARS-CoV2, the doses must be higher than those normally used for other diseases, which can cause serious adverse effects, some of which are fatal, especially in critically ill patients with COVID-19 (Das et al. 2020). Therefore, care should be taken with the safety aspects associated with the use of drugs to treat COVID-19.
Randomized clinical trials with a high degree of evidence (controlled, randomized, double-blind, and with adequate group size) of patients with COVID-19 will be needed before these drugs can be administered with confidence. Unfortunately, a single randomized controlled trial is not able to validate the use of any medication. Thus, there is a need for systematic reviews to monitor, synthesize, and update the literature data on the subject, generating reliable evidence for decision makers.

The main limitation of this systematic review is the very low quality of the evidence in most of the articles included, especially in relation to the methodology used. In addition, due to the scarcity of quality studies on the subject, articles with indirect results, non-randomized and non-blind studies were included, making the selection bias inevitable.

The data from this systematic review may provide assistance for the current urgent therapy demand for COVID-19. There are still no effective vaccines and antivirals to combat COVID-19, so it is necessary to monitor COVID-19 and reinforce the need to develop effective drugs and vaccines to prevent disease progression.

Acknowledgements The authors would like to thank the National Council for Scientific and Technological Development (CNPq), Coordination of Improvement of Higher-Level Personnel (CAPES Program CAPES/DFATD-88887.159028/2017-00, Program CAPES/ COFECUB-88881.370894/2019-01) and Foundation for Research Support of the State of Minas Gerais (FAPEMIG-APQ-00942-17). A. O. Andrade, A. A. Pereira, are Fellows of CNPq, Brazil (304818/20186 and 310911/2017-6, respectively).

\section{Declarations}

Conflict of interest The authors declare that they have no conflict of interest with this publication.

\section{References}

Cao B, Wang Y, Wen D, Liu W, Wang J, Fan G, et al. Trial of Lopinavirritonavir in adults hospitalized with severe Covid-19. N Engl J Med. 2020;382:1787-99. https://doi.org/10.1056/NEJMoa2001282.

Cascella M, Rajnik M, Cuomo A, Dulebohn SC, Di Napoli R. Features, evaluation and treatment coronavirus (COVID-19): StatPearls Publishing; 2020. PMid:32150360

Chen C, Qi F, Shi K, Li Y, Li J, Chen Y, Pan J, Zhou T, Lin X, Zhang J, Luo Y, Li X, Xia J. Thalidomide combined with low-dose glucocorticoid in the treatment of COVID-19 pneumonia. Preprints; 2020.

Choy KT, Wong AYL, Kaewpreedee P, Sia SF, Chen D, Hui KPY, Chu DKW, Chan MCW, Cheung PPH, Huang X, Peiris M, Yen HL. Remdesivir, lopinavir, emetine, and homoharringtonine inhibit SARS-CoV-2 replication in vitro. Antivir Res 2020;178. PMid: 32251767. https://doi.org/10.1016/j.antiviral.2020.104786, 104786.

Colson P, Rolain JM, Lagier JC, Brouqui P, Raoult D. Chloroquine and hydroxychloroquine as available weapons to fight COVID-19. Int J Antimicrob Agents 2020;55. PMid:32145363. https://doi.org/10. 1016/j.ijantimicag.2020.105932, 105932. 
Contini C, Nuzzo M Di, Barp N, Bonazza A, de Giorgio R, Tognon M, Rubino S. The novel zoonotic COVID-19 pandemic: an expected global health concern. J Infect Dev Ctries 2020;14:254-264. PMid: 32235085. https://doi.org/10.3855/jidc.12671.

Cortegiani A, Ingoglia G, Ippolito M, Giarratano A, Einav S. A systematic review on the efficacy and safety of chloroquine for the treatment of COVID-19. J Crit Care 2020. PMid:32173110. https://doi. org/10.1016/j.jcrc.2020.03.005, 57, 279, 283.

Das S, Bhowmick S, Tiwari S, Sen S. An updated systematic review of the therapeutic role of hydroxychloroquine in coronavirus disease19 (COVID-19). Clin Drug Investig. 2020;40:591-601. https://doi. org/10.1007/s40261-020-00927-1.

Deretic V, Timmins GS. BioRxiv: Azithromycin and ciprofloxacin have a chloroquine-like effect on respiratory epithelial cells; 2020.

van Doremalen N, Bushmaker T, Morris DH, Holbrook MG, Gamble A, Williamson BN, et al. Aerosol and surface stability of SARS-CoV-2 as compared with SARS-CoV-1. N Engl J Med. 2020;382:1564-7. https://doi.org/10.1056/NEJMc2004973.

Fantini J, Di Scala C, Chahinian H, Yahi N. Structural and molecular modelling studies reveal a new mechanism of action of chloroquine and hydroxychloroquine against SARS-CoV-2 infection. Int $\mathrm{J}$ Antimicrob Agents 2020. PMid:32251731. https://doi.org/10. 1016/j.ijantimicag.2020.105960, 55, 105960.

Gao J, Tian Z, Yang X. Breakthrough: chloroquine phosphate has shown apparent efficacy in treatment of COVID-19 associated pneumonia in clinical studies. Biosci Trends. 2020;14:72-3.

Gautret P, Lagier J-C, Parola P, Hoang VT, Meddeb L, Mailhe M, Doudier B, Courjon J, Giordanengo V, Vieira VE, Dupont HT, Honoré S, Colson P, Chabrière E, La Scola B, Rolain J-M, Brouqui P, Raoult D. Hydroxychloroquine and azithromycin as a treatment of COVID-19: results of an open-label non-randomized clinical trial. Int J Antimicrob Agents 2020:105949. PMid: 32205204. https://doi.org/10.1016/j.ijantimicag.2020.105949, 105949 .

Geleris J, Sun Y, Platt J, Zucker J, Baldwin M, Hripcsak G, et al. Observational study of hydroxychloroquine in hospitalized patients with Covid-19. N Engl J Med. 2020:NEJMoa2012410. https://doi. org/10.1056/NEJMoa2012410.

Holshue ML, DeBolt C, Lindquist S, Lofy KH, Wiesman J, Bruce H, Spitters C, Ericson K, Wilkerson S, Tural A, Diaz G, Cohn A, Fox LA, Patel A, Gerber SI, Kim L, Tong S, Lu X, Lindstrom S, Pallansch MA, Weldon WC, Biggs HM, Uyeki TM, Pillai SK. First case of 2019 novel coronavirus in the United States. N Engl J Med 2020;382:929-936. PMid:32004427. https://doi.org/10.1056/ NEJMoa2001191.

Hu BS, Gong JH, Sun J, Zhou JP. Exploring the epidemic transmission network of SARS in-out flow in mainland China. Chin Sci Bull. 2013;58:1818-31. https://doi.org/10.1007/s11434-012-5501-8.

Inciardi RM, Lupi L, Zaccone G, Italia L, Raffo M, Tomasoni D, et al. Cardiac involvement in a patient with coronavirus disease 2019 (COVID-19). JAMA Cardiol. 2020;5:819-24. https://doi.org/10. 1001/jamacardio.2020.1096

Juul S, Nielsen N, Bentzer P, Veroniki AA, Thabane L, Linder A, et al. Interventions for treatment of COVID-19: a protocol for a living systematic review with network meta-analysis including individual patient data (the LIVING project). Syst Rev. 2020;9:108. https://doi. org/10.1186/s13643-020-01371-0.

Kupferschmidt K, Cohen J. Race find COVID-19 treatments accelerates. 2020

Li T, Lu H, Zhang W. Clinical observation and management of COVID19 patients. Emerg Microbes Infect 2020;9:687-690. PMid: 32208840. https://doi.org/10.1080/22221751.2020.1741327.

Lim J, Jeon S, Shin HY, et al. Case of the index patient who caused tertiary transmission of COVID-19 infection in Korea: the application of lopinavir/ritonavir for the treatment of COVID-19 infected pneumonia monitored by quantitative RT-PCR. J Korean Med Sci. 2020.

Liu C, Zhou Q, Li Y, Garner LV, Watkins SP, Carter LJ, et al. Research and development on therapeutic agents and vaccines for COVID-19 and related human coronavirus diseases. ACS Cent Sci. 2020a;6: 315-31. https://doi.org/10.1021/acscentsci.0c00272.

Liu J, Cao R, Xu M, Wang X, Zhang H, Hu H, et al. Hydroxychloroquine, a less toxic derivative of chloroquine, is effective in inhibiting SARS-CoV-2 infection in vitro. Cell Discov. 2020b;6:16. https://doi.org/10.1038/s41421-020-0156-0.

Liu L, Gao J. Clinical characteristics of 51 patients discharged from hospital with COVID-19 in Chongqing, China. MedRxiv. 2020: 2020.02.20.20025536. https://doi.org/10.1101/2020.02.20. 20025536.

Liu W, Zhou P, Chen K, Ye Z, Liu F, Li X, et al. Efficacy and safety of antiviral treatment for COVID-19 from evidence in studies of SARS-CoV-2 and other acute viral infections: a systematic review and meta-analysis. Can Med Assoc J. 2020c;192:E734 LP-E744. https://doi.org/10.1503/cmaj.200647.

Madhav N, Oppenheim B, Gallivan M, Mulembakani P, Rubin E, Wolfe N. Pandemics: Risks, Impacts, and Mitigation. Dis. Control Priorities, Third Ed. (Volume 9) Improv. Heal. Reducing Poverty, The World Bank; 2017, p. 315-45. PMid:30212163. https://doi.org/ 10.1596/978-1-4648-0527-1_ch17.

Meo SA, Alhowikan AM, Khlaiwi TAL, Meo IM, Halepoto DM, Iqbal M, Usmani AM, Hajjar W, Ahmed N. Novel coronavirus 2019nCoV: Prevalence, biological and clinical characteristics comparison with SARS-CoV and MERS-CoV. Eur Rev Med Pharmacol Sci. 2020;24:2012-2019. PMid:32141570. https://doi.org/10. 26355/eurrev_202002_20379.

Mercuro NJ, Yen CF, Shim DJ, Maher TR, McCoy CM, Zimetbaum PJ, Gold HS. Risk of QT interval prolongation associated with use of hydroxychloroquine with or without concomitant azithromycin among hospitalized patients testing positive for coronavirus disease 2019 (COVID-19). JAMA Cardiol 2020. PMid:32356863. https:// doi.org/10.1001/jamacardio.2020.1834, 5, 1036, 1041.

Moher D, Shamseer L, Clarke M, Ghersi D, Liberati A, Petticrew M, Shekelle P, Stewart LA, Estarli M, Barrera ESA, MartínezRodríguez R, Baladia E, Agüero SD, Camacho S, Buhring K, Herrero-López A, Gil-González DM, Altman DG, Booth A, Chan AW, Chang S, Clifford T, Dickersin K, Egger M, Gøtzsche PC, Grimshaw JM, Groves T, Helfand M, Higgins J, Lasserson T, Lau J, Lohr K, Mc Gowan J, Mulrow C, Norton M, Page M, Sampson M, Schünemann H, Simera I, Summerskill W, Tetzlaff J, Trikalinos TA, Tovey D, Turner L, Whitlock E. Preferred Reporting Items for Systematic Review and Meta-Analysis Protocols (PRISMA-P) 2015 statement. Rev Esp Nutr Humana y Diet. 2016;20:148-160. PMid: 25554246. https://doi.org/10.1186/2046-4053-4-1.

OPAS/OMS Brasil. Folha informativa - COVID-19 (doença causada pelo novo coronavírus). 2020. [cited 2020 May 18]. Available from: https:/www.paho.org/bra/index.php?option=com_content\&view= article\&id=6101: covid19\&Itemid $=875$.

Paudel S, Dangal G, Chalise A, Bhandari TR, Dangal O. The coronavirus pandemic: What does the evidence show? J Nepal Health Res Counc. 2020;18:1-9. PMid:32335585. https://doi.org/10.33314/ jnhrc.v18i1.2596.

Reina J. Remdesivir, the antiviral hope against SARS-CoV-2. Rev Esp Quimioter Publ Of La Soc Esp Quimioter. 2020;33:176-9.

Rosenberg ES, Dufort EM, Udo T, Wilberschied LA, Kumar J, Tesoriero J, Weinberg P, Kirkwood J, Muse A, DeHovitz J, Blog DS, Hutton B, Holtgrave DR, Zucker HA. Association of treatment with hydroxychloroquine or azithromycin with in-hospital mortality in patients with COVID-19 in New York State. JAMA. 2020. PMid: 32392282. https://doi.org/10.1001/jama.2020.8630, 323, 2493, 2502. 
Sargiacomo C, Sotgia F, Lisanti MP. COVID-19 and chronological aging: senolytics and other anti-aging drugs for the treatment or prevention of corona virus infection. Albany NY: Aging; 2020.

Scuccimarri R, Sutton E, Fitzcharles M-A. Hydroxychloroquine: a potential ethical dilemma for rheumatologists during the COVID-19 pandemic. J Rheumatol. 2020;47:783-6.

Singh B, Ryan H, Kredo T, Chaplin M, Fletcher T. Chloroquine or hydroxychloroquine for prevention and treatment of COVID-19. Cochrane Database Syst Rev. 2020. https://doi.org/10.1002/ 14651858.CD013587.

Tang W, Cao Z, Han M, Wang Z, Chen J, Sun W, et al. Hydroxychloroquine in patients with mainly mild to moderate coronavirus disease 2019: open label, randomised controlled trial. BMJ. 2020;369:m1849. https://doi.org/10.1136/bmj.m1849.

Tobaiqy M, Qashqary M, Al-Dahery S, Mujallad A, Hershan AA, Kamal MA, et al. Therapeutic management of patients with COVID-19: a systematic review. Infect Prev Pract. 2020;2:100061. https://doi.org/ 10.1016/j.infpip.2020.100061.

Wang M, Cao R, Zhang L, Yang X, Liu J, Xu M, Shi Z, Hu Z, Zhong W, Xiao G. Remdesivir and chloroquine effectively inhibit the recently emerged novel coronavirus (2019-nCoV) in vitro. Cell Res 2020a;30:269-271. PMid:32020029. https://doi.org/10.1038/ s41422-020-0282-0.

Wang Z, Chen X, Lu Y, Chen F, Zhang W. Clinical characteristics and therapeutic procedure for four cases with 2019 novel Coronavírus pneumonia receiving combined Chinese and Western medicine treatment. Biosci Trends. 2020b;14:64-8. https://doi.org/10.5582/ bst.2020.01030.

Weston S, Haupt R, Logue J, Matthews K, Frieman M. FDA approved drugs with broad anti-coronaviral activity inhibit SARS-CoV-2 in vitro. BioRxiv. 2020:2020.03.25.008482. https://doi.org/10. 1101/2020.03.25.008482.

de Wit E, Feldmann F, Cronin J, Jordan R, Okumura A, Thomas T, Scott D, Cihlar T, Feldmann H. Prophylactic and therapeutic remdesivir (GS-5734) treatment in the rhesus macaque model of MERS-CoV infection. Proc Natl Acad Sci U S A 2020;117:6771-6776. PMid: 32054787. https://doi.org/10.1073/pnas.1922083117.

World Health Organization. Infection prevention and control of epidemic-and pandemic-prone acute respiratory infections in health care WHO Guidelines. WHO. 2014. [cited 2020 May 18]. Available from: https://www.who.int/publications/i/item/infectionprevention-and-control-of-epidemic-and-pandemic-prone-acuterespiratory-infections-in-health-care.

World Health Organization (WHO). Report of the WHO-China Joint Mission on Coronavirus Disease 2019 (COVID-19). 2020. [cited 2020 May 18]. Available from: https://www.who.int/publicationsdetail/report-of-the-who-china-joint-mission-on-coronavirusdisease-2019-(covid-19).

Xu X, Han M, Li T, Sun W, Wang D, Fu B, et al. Effective treatment of severe COVID-19 patients with tocilizumab. In: Effective treatment of severe COVID-19 patients with tocilizumab; 2020.

Yao X, Ye F, Zhang M, Cui C, Huang B, Niu P, Liu X, Zhao L, Dong E, Song C, Zhan S, Lu R, Li H, Tan W, Liu D. In vitro antiviral activity and projection of optimized dosing design of hydroxychloroquine for the treatment of severe acute respiratory syndrome coronavirus 2 (SARS-CoV-2). Clin Infect Dis 2020. PMid:32150618. https://doi. org/10.1093/cid/ciaa237, 71, 732, 739.

Yeh SH, Wang HY, Tsai CY, Kao CL, Yang JY, Liu HW, et al. Characterization of severe acute respiratory syndrome coronavirus genomes in Taiwan: molecular epidemiology and genome evolution. Proc Natl Acad Sci U S A. 2004;101:2542-7. https://doi.org/ 10.1073/pnas.0307904100.

Zhai P, Ding Y, Wu X, Long J, Zhong Y, Li Y. The epidemiology, diagnosis and treatment of COVID-19. Int J Antimicrob Agents 2020:105955. PMid:32234468. https://doi.org/10.1016/j. ijantimicag.2020.105955, 105955.

Publisher's note Springer Nature remains neutral with regard to jurisdictional claims in published maps and institutional affiliations. 